\title{
Assessment of bone density changes following two-jaw surgery using multidetector computed tomography: A pilot study
}

\author{
Youngjoo Lee ${ }^{\mathrm{a}}$ (1) \\ Jae Hyun Park ${ }^{\mathrm{b}, \mathrm{c}}$ \\ $\mathrm{Na}$-Young Chang ${ }^{\mathrm{d}}$ \\ Mi-Young Lee ${ }^{\mathrm{e}}$ \\ Bong Chul Kim ${ }^{f}$ \\ Hye Young Seo ${ }^{g}$ \\ Utkarsh Mangal $^{\mathrm{h}}$ \\ Jong-Moon Chae ${ }^{\mathrm{b}, \mathrm{d}}$ (1)
}

${ }^{a}$ Department of Orthodontics, Wonkwang University College of Dentistry, lksan, Korea

${ }^{b}$ Postgraduate Orthodontic Program, Arizona School of Dentistry and Oral Health, A.T. Still University, Mesa, AZ, USA

'Graduate School of Dentistry, Kyung Hee University, Seoul, Korea

${ }^{\mathrm{d}}$ Department of Orthodontics, Wonkwang Dental Research Institute, Wonkwang University College of Dentistry, lksan, Korea

'Department of Orthodontics, Seoul National University Gwanak Dental Hospital, Seoul, Korea

'Department of Oral and Maxillofacial Surgery, Wonkwang University Daejeon Dental Hospital, Daejeon, Korea

${ }^{9}$ School of Big Data and Financial Statistics, Wonkwang University College of Natural Sciences, lksan, Korea

${ }^{\text {h}}$ Department of Orthodontics, Yonsei University College of Dentistry, Seoul, Korea

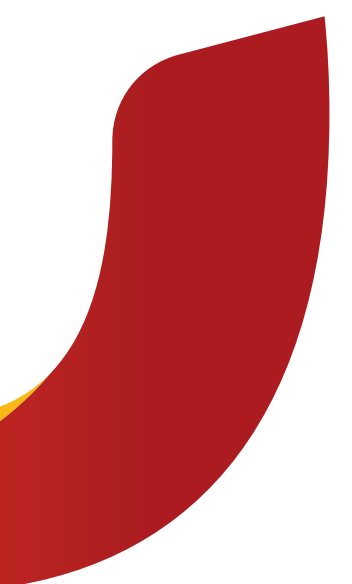

Objective: The aim of this retrospective study was to evaluate the pre- and postsurgical bone densities at alveolar and extra-alveolar sites following twojaw orthognathic surgery. Methods: The sample consisted of 10 patients (mean age, 23.2 years; range, 18.0-27.8 years; 8 males, 2 females) who underwent two-jaw orthognathic surgery. A three-dimensional imaging program (Invivo 5) was used with multidetector computed tomography images taken preand postoperatively (obtained $32.3 \pm 6.0$ days before surgery and $5.8 \pm 2.6$ days after surgery, respectively) for the measurement of bone densities at the following sites: (1) alveolar bone in the maxilla and mandible, (2) extra-alveolar sites, such as the top of the head, menton (Me), condyle, and the fourth cervical vertebrae (C4). Results: When pre- and postsurgical bone densities were compared, an overall tendency of decrease in bone density was noted. Statistically significant reductions were observed in the densities of cancellous bone at several areas of the maxillary alveolar bone; cortical and cancellous bone in most areas of the mandibular alveolar bone; cortical bone in Me; and cancellous bone in C4. There was no statistically significant difference in bone density in relation to the depth of the alveolar bone. In a comparison of the bone densities between groups with and without genioplasty, there was almost no statistically significant difference. Conclusions: Accelerated tooth movement following orthognathic surgery may be confirmed with reduced bone density. In addition, this study could offer insights into bone metabolism changes following orthognathic surgery, providing direction for further investigations in this field.

[Korean J Orthod 2020;50(3):157-169]

Key words: Bone density, Orthognathic surgery, Multidetector computed tomography, Regional and systemic acceleratory phenomena

Received August 29, 2019; Revised November 6, 2019; Accepted December 27, 2019.

Corresponding author: Jong-Moon Chae.

Professor, Department of Orthodontics, Wonkwang University Daejeon Dental Hospital, 77 Dunsan-ro, Seo-gu, Daejeon 35233, Korea.

Tel +82-42-366-1103 e-mail jongmoon@wku.ac.kr

How to cite this article: Lee Y, Park JH, Chang NY, Lee MY, Kim BC, Seo HY, Mangal U, Chae JM. Assessment of bone density changes following two-jaw surgery using multidetector computed tomography: A pilot study. Korean J Orthod 2020;50:157-169.

(C) 2020 The Korean Association of Orthodontists.

This is an Open Access article distributed under the terms of the Creative Commons Attribution Non-Commercial License (http://creativecommons.org/licenses/by-nc/4.0) which permits unrestricted non-commercial use, distribution, and reproduction in any medium, provided the original work is properly cited. 


\section{INTRODUCTION}

Acceleration of orthodontic tooth movement is a major issue due to patient demand for shorter orthodontic treatment time. Patients as well as clinicians benefit greatly from shorter treatment durations. Since the late 1950s, several surgical techniques, including corticotomy, have been suggested to reduce the duration of orthodontic treatment by facilitating tooth movement. ${ }^{1}$ These techniques are principally based on the regional acceleratory phenomenon (RAP).

RAP, which was first described by Frost, ${ }^{2-4}$ refers to the acceleration of bone metabolism following the application of a noxious stimuli, with a resultant increase in the concentration of bone metabolic products (osteoclasts and osteoblasts) during the healing process of the damaged structure. RAP can be initiated by injury, surgical operation, or even by orthodontic tooth movement. ${ }^{5}$ RAP results in a temporary decrease in regional bone density and an increase in remodeling.

When Mueller et al. ${ }^{6}$ investigated the effect of localized trauma in rats in 1991, they discovered that a local defect in the tibia caused cancellous bone density changes in distant sites, suggesting that other skeletal locations, such as the femur and lumbar vertebrae could be affected as well. Furthermore, the local defect induced a systemic impact on bone metabolism, while local repair, either of the bone or of the bone marrow, led to the release of humoral factors that acted systemically via the circulation. This process was termed "systemic acceleratory phenomenon (SAP)".

SAP was also thought to occur as a result of orthognathic surgery. Liou et al. ${ }^{7}$ confirmed the occurrence of SAP with orthognathic surgery, noting an increase in the serum levels of alkaline phosphatase (ALP, a biomarker for osteoblastic activity) and C-terminal telopeptide of type 1 collagen (1CTP, a biomarker for osteoclastic activity) following orthognathic surgery. RAPs such as tooth mobility corresponded significantly with changes in ICTP, but not with changes in ALP.

Orthodontic tooth movement and RAP have been very well researched from the biological and histological points of view, but there is little radiological research addressing this topic. ${ }^{8-10}$ Moreover, most studies focused mainly on RAP, so radiological observations concerning SAP are scarce. To date, no study has investigated the exact dimensional changes in the bone density of patients undergoing orthognathic surgery.

Therefore, this study aimed to assess the regional and systemic changes in bone density following orthognathic surgery. To achieve this, bone density was assessed in alveolar bone and at other areas, including the fourth cervical vertebrae (C4), cranial bone, mandibular condyle, and menton (Me), using multidetector computed tomography (MDCT).

\section{MATERIALS AND METHODS}

\section{Sample size calculation}

A power analysis using $G^{*}$ Power software ver. 3.1.9.2 (Franz Faul; Christian-Albrechts-Universitat, Kiel, Germany) was performed to estimate the power of the analysis using a sample size of 10 . With a two-tail model, effect size of 0.8 , and a total sample size of 10 , the estimated $\alpha$ error probability was $0.05, \beta$ error probability was 0.4 , and power was 0.6 .

\section{Subjects, eligibility criteria, and computed tomography}

The sample consisted of 10 sets of pre- and postsurgical medical grade computed tomography (CT) scans from skeletal Class 111 patients who underwent twojaw orthognathic surgery at the Wonkwang University Daejeon Dental Hospital in Daejeon, Korea. All surgical operations were performed by the same surgeon.

The sample distribution is shown in Table 1. The sample consisted of a total of 10 patients (mean age, $23.2 \pm$ 3.1 years; range, 18.0-27.8 years), which included eight men (mean age, $23.3 \pm 3.1$ years) and two women (mean age, $22.8 \pm 4.0$ years). All patients had undergone intraoral vertical ramus osteotomy (IVRO) for mandibular setback and Le Fort 1 osteotomy for maxillary advancement. Among them, six patients had previously undergone genioplasty.

The inclusion criteria were as follows: (1) diagnosed as showing a Class 111 skeletal relationship; (2) received two-jaw orthognathic surgery; (3) CT scans were taken at least twice: before surgery ( $\mathrm{T} 1)$ and within 2 months after surgery (T2); and (4) presenting with no craniofacial abnormalities.

CT images were taken with multidetector CT (Siemens Somatom Definition Edge; Siemens Healthcare GmbH, Erlangen, Germany) at the Eulji University Medical Hospital, and were stored on a CD and brought to Wonkwang University Daejeon Dental Hospital. Before surgery, all patients provided informed consent to the Department of Oral and Maxillofacial Surgery for MDCT examinations to acquire information necessary for surgery and evaluation of the surgery. The following radiologic parameters were used in the MDCT examinations: $120 \mathrm{kV}$; $60 \mathrm{mAs}$; exposure time, 1,000 ms; matrix size, $512 \times 512$; a field of view, $200 \mathrm{~mm}$ in diameter. The T1 images were obtained $32.3 \pm 6.0$ days before surgery, and the T2 images were obtained $5.8 \pm 2.6$ days after surgery. The CT data were saved in digital imaging and communications in medicine (DICOM) files, and Invivo 5 software ver. 5.5.2 (Anatomage; San Jose, CA, USA) was used to analyze the DICOM data to generate the quantitative measurements. 
Institutional Review Board approval to conduct this study was granted by Wonkwang University Daejeon Dental Hospital (number WKD IRB W1811/001-001) in Daejeon, Korea.

\section{Study design}

After storing CT images with slice thicknesses of 0.75 $\mathrm{mm}$ into a personal computer, Invivo 5 software (Anatomage) was used to reconstruct and reorient the DICOM files. All images were reoriented by using a Frankfurt horizontal plane (Figure 1).

The bone density was measured at two different areas to investigate RAP and SAP: (1) alveolar sites in the maxilla and mandible and (2) extra-alveolar sites for the investigation of bone density changes in distant areas.

To measure the alveolar bone densities, two different reorientation steps were used in each arch (Figure 2). The alveolar bone density was measured at four sites: (1) between the central and lateral incisors; (2) between the first and second premolars or between the canines and remaining premolars when premolar extraction was performed; (3) between the second and first molars; and (4)

Table 1. Sample distribution

\begin{tabular}{lrr}
\hline \multicolumn{1}{c}{ Variable } & $\begin{array}{c}\text { Mean } \pm \text { standard } \\
\text { deviation }\end{array}$ & Range \\
\hline Age at surgery (year) & & \\
Overall (n = 10) & $23.2 \pm 3.1$ & $18.0-27.8$ \\
Male (n=8) & $23.3 \pm 3.1$ & $18.0-27.8$ \\
Female ( $\mathrm{n}=2)$ & $22.8 \pm 4.0$ & $20.0-25.7$ \\
T1 image (day) & $32.3 \pm 6.0$ & $23.0-42.0$ \\
T2 image (day) & $5.8 \pm 2.6$ & $3.0-11.0$ \\
Date difference between & $38.1 \pm 6.5$ & $27.0-46.0$ \\
T1 and T2 CT image (day) & & \\
\hline
\end{tabular}

T1, Before surgery; T2, after surgery; CT, computed tomography. at the maxillary tuberosity or mandibular retromolar pad (Figure 3). For extra-alveolar measurements, four areas were selected as follows: (1) top of the head, (2) Me, (3) left condyle, and (4) C4 (Figures 4-7). The bone density was measured in Hounsfield units (HU) at every site.

\section{Statistical analysis}

One investigator (Y.L.) performed all the measurements on the 10 subjects. To test the reliability of the measurements, four subjects were randomly selected for re-measurement at least two weeks after the initial measurement. The intra-class correlation coefficients (ICCs) showed excellent test-retest reliability of the bone density measurement $(\mathrm{ICC}=0.99)$. A paired $t$-test on the data from the second round showed no significant difference between the measured values $(p=0.123$ and $p=0.978$ ).

Means and standard deviations were calculated for each parameter. A Shapiro-Wilk normality test was performed and a nonparametric test was performed when the normality was not satisfied. T1 and T2 data were analyzed by using the paired sample $t$-test or the nonparametric Wilcoxon signed rank tests. Additionally, an independent-samples Kruskal-Wallis test was performed to examine the difference between depths in the alveolar area. Finally, an independent sample t-test or a MannWhitney test was performed to determine the difference between the patients who had undergone genioplasty and those who had not. IBM SPSS ver. 25 (IBM Corp., Armonk, NY, USA) was used for statistical analyses, and statistical significance was based on a $p$-value of $<0.05$.

\section{RESULTS}

The mean values of alveolar bone density were compared between $\mathrm{T} 1$ and $\mathrm{T} 2$. Statistical differences were found in more sites in the mandible than in the maxilla. The cancellous bone dimensions showed predominant statistical significance in comparison with those of the
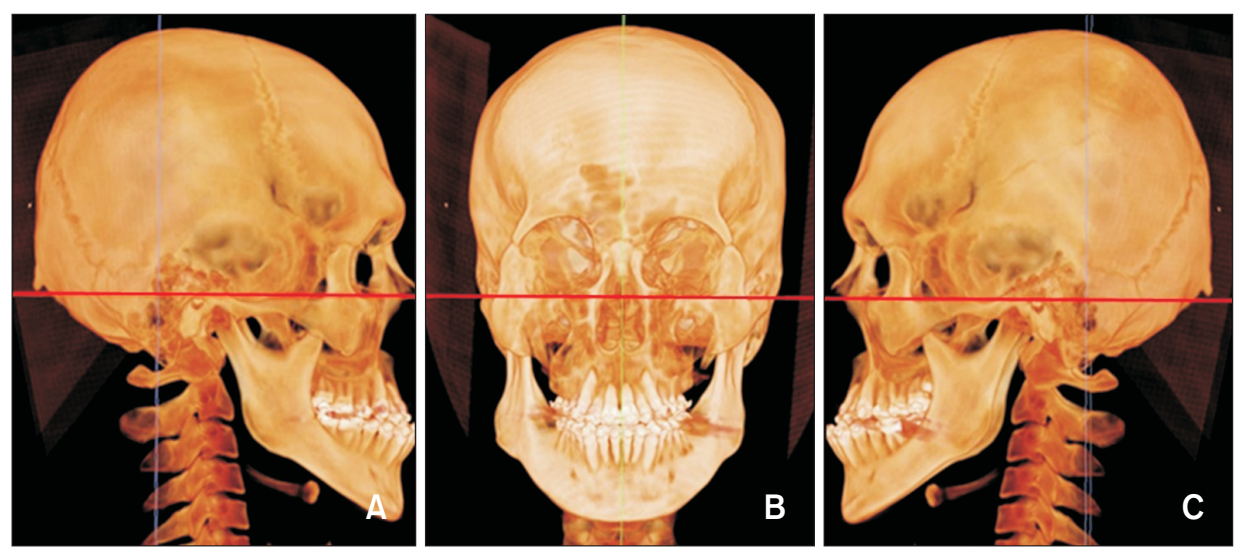

Figure 1. Frankfurt horizontal (FH) plane orientation of computed tomography images. All images were reoriented to the $\mathrm{FH}$ plane using both porions and the right orbitale on the red line. A, Right lateral view. B, Frontal view. C, Left lateral view. 


\section{KJO}
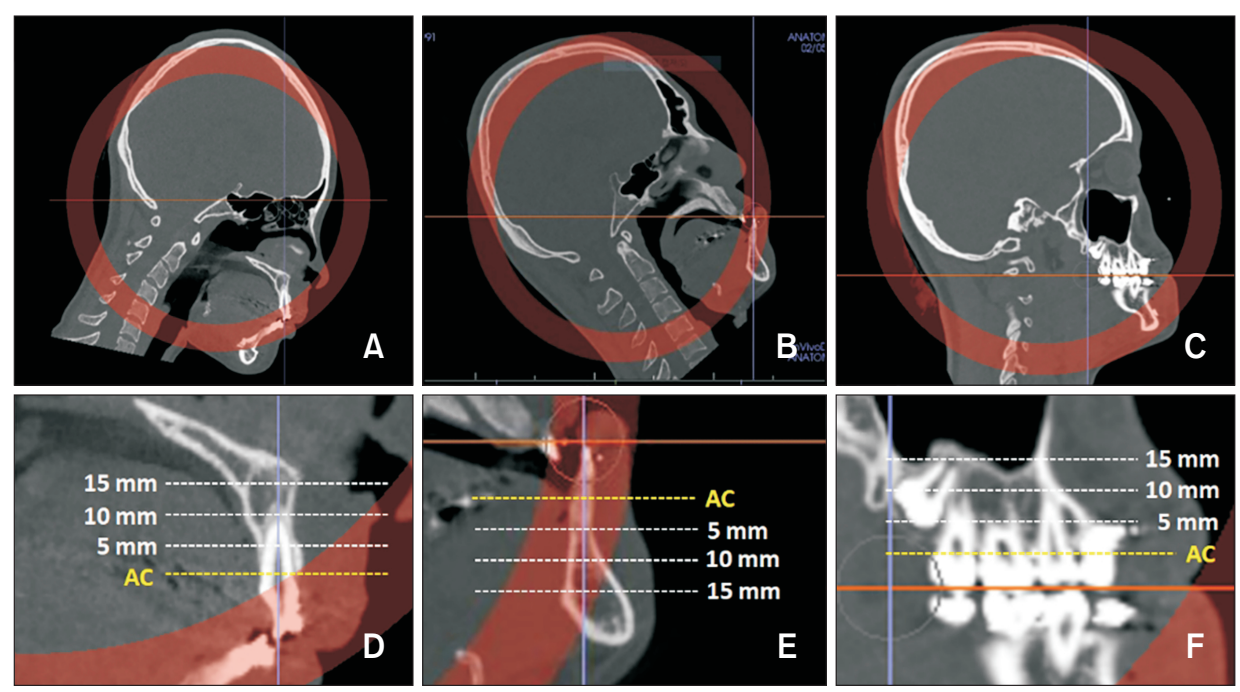

Figure 2. Reorientation and measurement of alveolar bone density in the sagittal views. A, B, Computed tomography images were reoriented to align the axis of the left maxillary and mandibular central incisors perpendicular to the horizontal plane. C, Posterior occlusal plane was aligned so it was parallel to the horizontal plane. D-F, Bone densities were measured at the alveolar crest ( $A C$, yellow line) and at each depth (dotted lines; 5 , $10,15 \mathrm{~mm}$ from $\mathrm{AC}$ ).
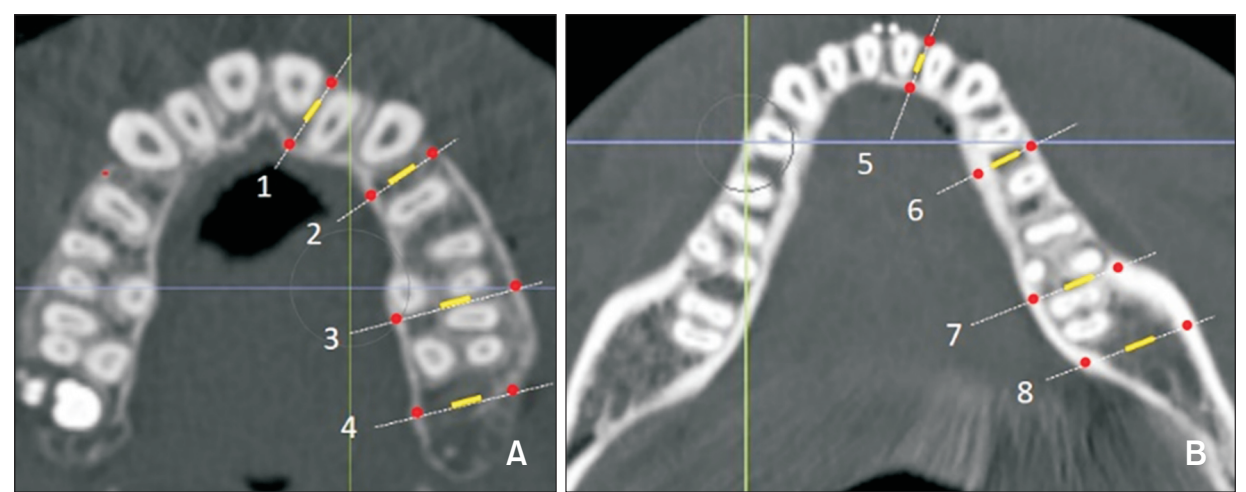

Figure 3. Measurement sites in the alveolar bone. A, B, Measurement sites in the maxilla and mandible. 1, 5: Between central and lateral incisors. 2, 6: Between the first and second premolars or between canines and remaining premolars when premolar extraction was performed. 3, 7: Between the second and first molars. 4: Maxillary tuberosity. 8: Retromolar pad. All measurements were acquired from the left side of the arch. Measurement areas of cortical and cancellous bone are shown as red dots and a yellow line, respectively.
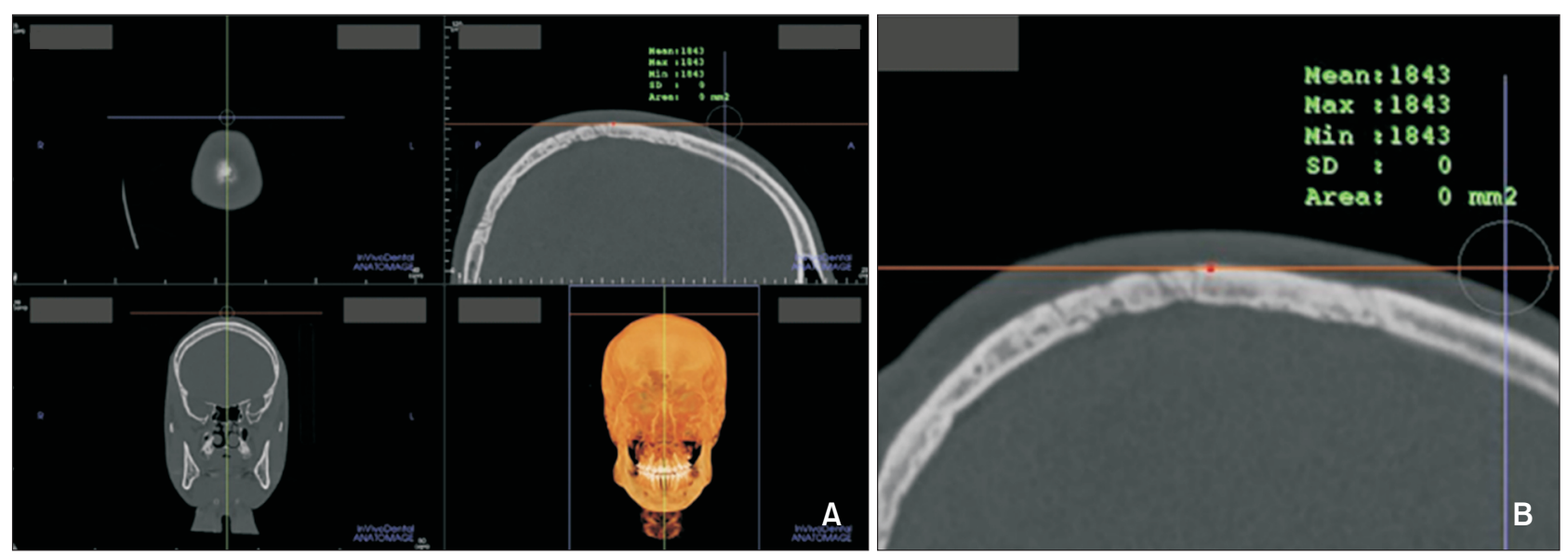

Figure 4. Bone density measurement at the top point of the head. A, The most superior point was selected in the midsagittal view. B, Magnified view of the measurement site. 

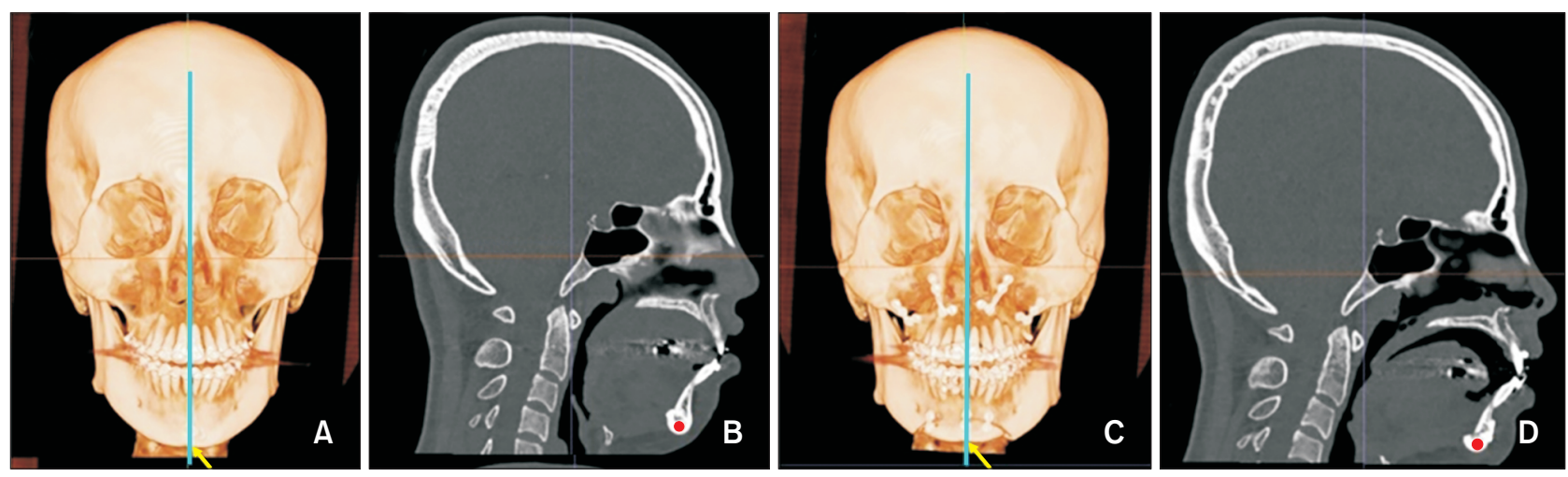

Figure 5. Bone density measurement at menton (Me). A, Presurgical image, the blue line represents the vertical slice drawn over Me (yellow arrow). B, Presurgical sagittal slice at Me (red dot). C, Postsurgical image. D, Postsurgical sagittal slice at Me (red dot).
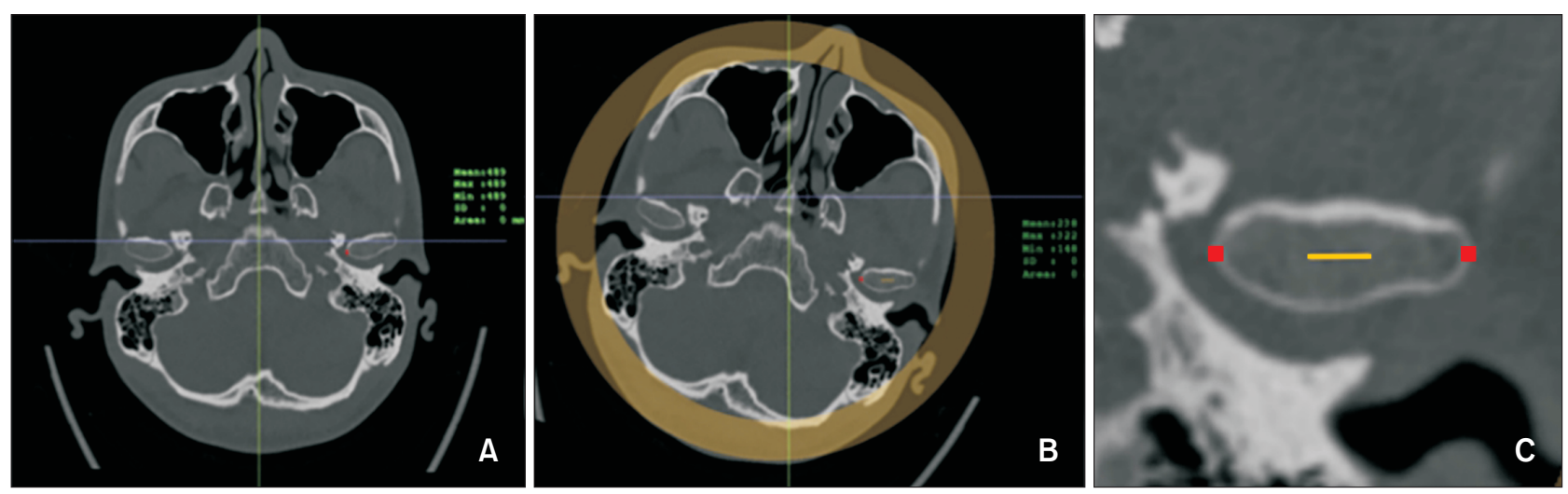

Figure 6. Bone density measurement at the condyle. A, The axial slice with the largest mediolateral diameter of the mandibular condyle was selected. B, The condyle was re-oriented as the most medial and lateral points aligned in a horizontal line. C, Cortical bone densities at the medial and lateral points were measured (red dots), and cancellous bone density was measured in the middle $5 \mathrm{~mm}$ upon the line connecting the medial and lateral points.
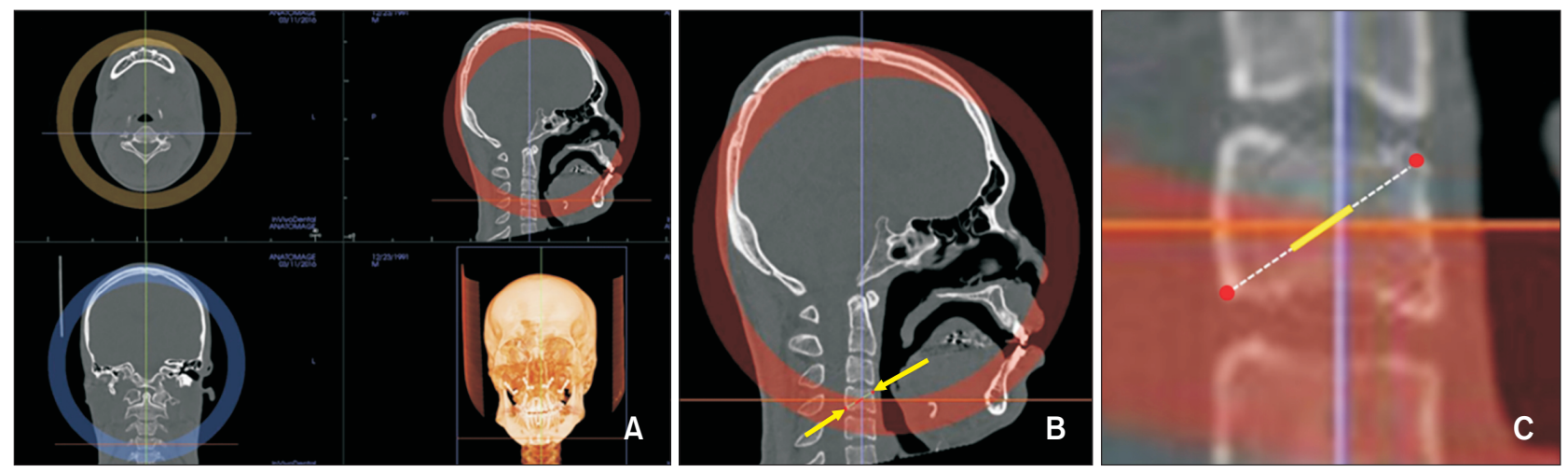

Figure 7. Bone density measurements for the fourth cervical vertebrae (C4). A, Orientation of computed tomography images for C4. B, Measurement sites for C4 (yellow arrow). C, Magnified view of C4. Cortical bone densities were measured at the most anterosuperior and posteroinferior red points and cancellous bone density was measured in the middle $5 \mathrm{~mm}$ of the line (yellow) connecting the two red points. 
Table 2. Comparison of alveolar bone density (HU) in the maxilla between $\mathrm{T} 1$ and $\mathrm{T} 2$ measurements

\begin{tabular}{|c|c|c|c|c|c|c|c|}
\hline & Site & & T1 & T2 & $\Delta(\mathrm{T} 2-\mathrm{T} 1)$ & $p$-value & Number \\
\hline \multirow[t]{9}{*}{ Tuberosity } & $5 \mathrm{~mm}$ & $\mathrm{BC}$ & $763.0 \pm 138.4$ & $746.8 \pm 102.2$ & $-16.2 \pm 80.7$ & 0.677 & 5 \\
\hline & & $\mathrm{LC}$ & $919.8 \pm 117.8$ & $892.2 \pm 114.3$ & $-27.6 \pm 45.6$ & 0.247 & 5 \\
\hline & & Can & $212.2 \pm 66.9$ & $120.0 \pm 87.3$ & $-92.2 \pm 57.4$ & $0.023^{*}$ & 5 \\
\hline & $10 \mathrm{~mm}$ & $\mathrm{BC}^{\dagger}$ & $1,024.5 \pm 277.0$ & $1,034.5 \pm 234.2$ & $10.0 \pm 107.2$ & $>0.999$ & 4 \\
\hline & & $\mathrm{LC}$ & $1,285.5 \pm 215.8$ & $1,215.5 \pm 206.6$ & $-70.0 \pm 11.9$ & $0.001^{* *}$ & 4 \\
\hline & & Can & - & - & - & - & \\
\hline & $15 \mathrm{~mm}$ & $\mathrm{BC}$ & - & - & - & - & 0 \\
\hline & & $\mathrm{LC}$ & - & - & - & - & 0 \\
\hline & & Can & - & - & - & - & 0 \\
\hline \multirow[t]{9}{*}{ Molar } & $5 \mathrm{~mm}$ & $\mathrm{BC}$ & $1,059.2 \pm 138.1$ & $1,053.2 \pm 138.3$ & $-6.0 \pm 37.9$ & 0.648 & 9 \\
\hline & & $\mathrm{LC}$ & $1,198.0 \pm 295.7$ & $1,168.2 \pm 282.8$ & $-29.8 \pm 65.1$ & 0.185 & 10 \\
\hline & & Can & $452.5 \pm 247.0$ & $394.4 \pm 190.3$ & $-58.1 \pm 71.7$ & 0.055 & 8 \\
\hline & $10 \mathrm{~mm}$ & $\mathrm{BC}$ & $1,243.1 \pm 346.9$ & $1,154.4 \pm 310.9$ & $-88.8 \pm 133.7$ & 0.103 & 8 \\
\hline & & $\mathrm{LC}$ & $1,407.8 \pm 249.5$ & $1,392.1 \pm 172.5$ & $-15.7 \pm 131.1$ & 0.714 & 10 \\
\hline & & Can & $204.5 \pm 316.1$ & $115.0 \pm 87.7$ & $-89.5 \pm 228.4$ & 0.678 & 2 \\
\hline & $15 \mathrm{~mm}$ & $\mathrm{BC}^{\dagger}$ & $1,540.0 \pm 156.1$ & $1,460.5 \pm 109.6$ & $-79.5 \pm 72.5$ & 0.109 & 4 \\
\hline & & $\mathrm{LC}$ & $1,110.4 \pm 179.9$ & $1,113.0 \pm 193.5$ & $2.6 \pm 78.7$ & 0.945 & 5 \\
\hline & & Can & 174.0 & 277.0 & 103.0 & - & 1 \\
\hline \multirow[t]{9}{*}{ Premolar } & $5 \mathrm{~mm}$ & $\mathrm{BC}$ & $1,225.7 \pm 181.3$ & $1,222.0 \pm 261.7$ & $-3.7 \pm 126.9$ & 0.929 & 10 \\
\hline & & $\mathrm{LC}$ & $1,246.1 \pm 163.1$ & $1,274.6 \pm 178.5$ & $28.5 \pm 83.0$ & 0.306 & 10 \\
\hline & & Can & $464.7 \pm 271.2$ & $372.4 \pm 251.0$ & $-92.3 \pm 94.4$ & $0.013^{*}$ & 10 \\
\hline & $10 \mathrm{~mm}$ & $\mathrm{BC}^{\dagger}$ & $1,297.5 \pm 387.9$ & $1,271.8 \pm 364.1$ & $-25.7 \pm 64.1$ & 0.203 & 10 \\
\hline & & $\mathrm{LC}$ & $1,353.1 \pm 203.7$ & $1,377.2 \pm 219.8$ & $24.1 \pm 117.0$ & 0.531 & 10 \\
\hline & & Can & $380.6 \pm 261.4$ & $292.4 \pm 236.0$ & $-88.1 \pm 55.7$ & $0.006^{* *}$ & 7 \\
\hline & $15 \mathrm{~mm}$ & $\mathrm{BC}^{\dagger}$ & $1,639.6 \pm 201.3$ & $1,589.9 \pm 174.5$ & $-49.8 \pm 116.7$ & 0.263 & 8 \\
\hline & & $\mathrm{LC}$ & $1,220.4 \pm 319.8$ & $1,197.9 \pm 355.2$ & $-22.6 \pm 75.4$ & 0.458 & 7 \\
\hline & & $\mathrm{Can}^{\dagger}$ & $220.5 \pm 294.9$ & $218.0 \pm 253.1$ & $-2.5 \pm 41.7$ & 0.655 & 2 \\
\hline \multirow[t]{9}{*}{ Incisal } & $5 \mathrm{~mm}$ & $\mathrm{BC}^{\dagger}$ & $1,101.3 \pm 335.8$ & $1,061.5 \pm 286.6$ & $-39.8 \pm 92.9$ & 0.093 & 10 \\
\hline & & $\mathrm{LC}^{\dagger}$ & $1,068.1 \pm 268.4$ & $1,040.1 \pm 258.0$ & $-28.0 \pm 154.1$ & 0.285 & 10 \\
\hline & & Can & $568.8 \pm 292.4$ & $452.8 \pm 255.2$ & $-116.0 \pm 135.7$ & $0.046^{*}$ & 8 \\
\hline & $10 \mathrm{~mm}$ & $\mathrm{BC}$ & $1,043.6 \pm 286.4$ & $1,044.2 \pm 324.3$ & $0.6 \pm 120.6$ & 0.988 & 10 \\
\hline & & $\mathrm{LC}^{\dagger}$ & $1,303.1 \pm 177.0$ & $1,271.7 \pm 207.9$ & $-31.4 \pm 142.3$ & 0.333 & 10 \\
\hline & & Can & $498.6 \pm 201.7$ & $416.2 \pm 224.1$ & $-82.3 \pm 119.7$ & 0.073 & 9 \\
\hline & $15 \mathrm{~mm}$ & $\mathrm{BC}^{\dagger}$ & $1,360.1 \pm 215.1$ & $1,309.9 \pm 272.6$ & $-50.2 \pm 70.0$ & 0.059 & 10 \\
\hline & & LC & $1,308.4 \pm 165.3$ & $1,273.0 \pm 152.5$ & $-35.4 \pm 67.7$ & 0.132 & 10 \\
\hline & & Can & $235.6 \pm 120.1$ & $195.1 \pm 112.5$ & $-40.4 \pm 39.9$ & $0.016^{*}$ & 9 \\
\hline
\end{tabular}

Values are presented as mean \pm standard deviation or number only when only one measurement was possible.

Some measurements could not be obtained due to adjacent anatomic structures (i.e., third molar, maxillary sinus) or other objects (i.e., orthodontic miniscrew).

HU, Hounsfield units; T1, before surgery; T2, after surgery; BC, buccal cortical bone; LC, lingual cortical bone; Can, cancellous bone.

${ }^{*} p<0.05,{ }^{* *} p<0.01$.

The paired sample $t$-tests or ${ }^{\dagger}$ Wilcoxon signed rank tests were performed $(\alpha=0.05)$. 
Table 3. Comparison of alveolar bone density (HU) in the mandible between T1 and T2 measurements

\begin{tabular}{|c|c|c|c|c|c|c|c|}
\hline \multicolumn{3}{|c|}{ Site } & \multirow{2}{*}{$\begin{array}{c}\text { T1 } \\
1,695.6 \pm 337.5\end{array}$} & \multirow{2}{*}{$\begin{array}{c}\text { T2 } \\
1,640.5 \pm 269.0\end{array}$} & \multirow{2}{*}{$\begin{array}{c}\Delta(\mathbf{T} 2-\mathrm{T} 1) \\
-55.1 \pm 94.0\end{array}$} & \multirow{2}{*}{$\begin{array}{c}\boldsymbol{p} \text {-value } \\
0.093\end{array}$} & \multirow{2}{*}{$\begin{array}{c}\text { Number } \\
10\end{array}$} \\
\hline Retromolar pad & $5 \mathrm{~mm}$ & $\mathrm{BC}^{\dagger}$ & & & & & \\
\hline & & $\mathrm{LC}$ & $1,196.6 \pm 361.7$ & $1,145.7 \pm 329.9$ & $-50.9 \pm 38.4$ & $0.002^{* *}$ & 10 \\
\hline & & Can & $219.1 \pm 130.9$ & $150.8 \pm 105.5$ & $-68.3 \pm 91.1$ & $0.042^{*}$ & 10 \\
\hline & $10 \mathrm{~mm}$ & $\mathrm{BC}$ & $1,904.1 \pm 93.8$ & $1,824.8 \pm 146.7$ & $-79.3 \pm 129.2$ & 0.084 & 10 \\
\hline & & $\mathrm{LC}$ & $1,759.1 \pm 74.3$ & $1,661.3 \pm 62.0$ & $-97.8 \pm 110.7$ & $0.021^{*}$ & 10 \\
\hline & & Can & $245.9 \pm 127.6$ & $175.4 \pm 103.4$ & $-70.5 \pm 57.6$ & $0.004^{* *}$ & 10 \\
\hline & $15 \mathrm{~mm}$ & $\mathrm{BC}$ & $1,919.8 \pm 102.4$ & $1,904.3 \pm 105.7$ & $-15.5 \pm 32.3$ & 0.163 & 10 \\
\hline & & $\mathrm{LC}$ & $1,723.8 \pm 102.6$ & $1,700.0 \pm 100.9$ & $-23.8 \pm 84.3$ & 0.395 & 10 \\
\hline & & Can & $159.4 \pm 99.3$ & $102.3 \pm 109.4$ & $-57.1 \pm 62.4$ & $0.018^{*}$ & 10 \\
\hline \multirow[t]{9}{*}{ Molar } & $5 \mathrm{~mm}$ & $\mathrm{BC}$ & $1,575.5 \pm 95.9$ & $1,541.6 \pm 121.9$ & $-33.9 \pm 70.9$ & 0.165 & 10 \\
\hline & & $\mathrm{LC}$ & $1,660.9 \pm 123.1$ & $1,577.8 \pm 113.3$ & $-83.1 \pm 65.9$ & $0.003^{* *}$ & 10 \\
\hline & & Can & $548.9 \pm 172.4$ & $449.7 \pm 147.4$ & $-99.2 \pm 72.2$ & $0.002^{* *}$ & 10 \\
\hline & $10 \mathrm{~mm}$ & $\mathrm{BC}$ & $1,829.2 \pm 103.6$ & $1,749.2 \pm 166.2$ & $-80.0 \pm 86.2$ & $0.017^{*}$ & 10 \\
\hline & & $\mathrm{LC}$ & $1,800.1 \pm 88.0$ & $1,750.3 \pm 127.3$ & $-49.8 \pm 80.4$ & 0.082 & 10 \\
\hline & & Can & $188.7 \pm 156.3$ & $110.9 \pm 149.0$ & $-77.8 \pm 72.9$ & $0.008^{* *}$ & 10 \\
\hline & $15 \mathrm{~mm}$ & $\mathrm{BC}$ & $1,886.8 \pm 89.7$ & $1,820.7 \pm 102.9$ & $-66.1 \pm 105.6$ & 0.079 & 10 \\
\hline & & LC & $1,796.2 \pm 121.6$ & $1,757.6 \pm 103.9$ & $-38.6 \pm 61.5$ & 0.078 & 10 \\
\hline & & Can & $111.9 \pm 157.0$ & $71.4 \pm 136.8$ & $-40.5 \pm 30.6$ & $0.002^{* *}$ & 10 \\
\hline \multirow[t]{9}{*}{ Premolar } & $5 \mathrm{~mm}$ & $\mathrm{BC}$ & $1,435.5 \pm 148.6$ & $1,369.2 \pm 131.7$ & $-66.3 \pm 53.7$ & $0.004^{* *}$ & 10 \\
\hline & & $\mathrm{LC}$ & $1,589.5 \pm 112.8$ & $1,505.9 \pm 143.3$ & $-83.6 \pm 88.7$ & $0.015^{*}$ & 10 \\
\hline & & Can & $183.9 \pm 142.8$ & $132.4 \pm 123.1$ & $-51.5 \pm 47.4$ & $0.007^{* *}$ & 10 \\
\hline & $10 \mathrm{~mm}$ & $\mathrm{BC}$ & $1,573.2 \pm 175.6$ & $1,544.2 \pm 118.0$ & $-29.0 \pm 167.6$ & 0.598 & 10 \\
\hline & & LC & $1,782.5 \pm 115.6$ & $1,700.3 \pm 107.0$ & $-82.2 \pm 105.1$ & $0.035^{*}$ & 10 \\
\hline & & $\mathrm{Can}^{\dagger}$ & $211.9 \pm 136.3$ & $137.2 \pm 135.0$ & $-74.7 \pm 88.1$ & $0.009^{* *}$ & 10 \\
\hline & $15 \mathrm{~mm}$ & $\mathrm{BC}$ & $1,852.0 \pm 139.8$ & $1,758.9 \pm 133.2$ & $-83.1 \pm 101.7$ & $0.030^{*}$ & 10 \\
\hline & & $\mathrm{LC}$ & $1,883.1 \pm 106.4$ & $1,804.8 \pm 121.5$ & $-78.3 \pm 99.3$ & $0.034^{*}$ & 10 \\
\hline & & Can & $156.5 \pm 185.2$ & $89.8 \pm 150.9$ & $-66.7 \pm 50.3$ & $0.002^{* *}$ & 10 \\
\hline \multirow[t]{9}{*}{ Incisal } & $5 \mathrm{~mm}$ & $\mathrm{BC}$ & $764.6 \pm 208.1$ & $713.5 \pm 214.9$ & $-51.1 \pm 55.9$ & $0.018^{*}$ & 10 \\
\hline & & LC & $1,051.4 \pm 159.6$ & $984.7 \pm 204.4$ & $-66.7 \pm 90.1$ & $0.044^{*}$ & 10 \\
\hline & & Can & $510.0 \pm 204.5$ & $407.3 \pm 219.2$ & $-102.7 \pm 73.5$ & $0.002^{* *}$ & 10 \\
\hline & $10 \mathrm{~mm}$ & $\mathrm{BC}$ & $1,142.0 \pm 358.7$ & $1,129.6 \pm 383.9$ & $-12.4 \pm 51.6$ & 0.467 & 10 \\
\hline & & $\mathrm{LC}^{\dagger}$ & $1,464.7 \pm 253.3$ & $1,446.3 \pm 264.7$ & $-18.4 \pm 37.8$ & 0.241 & 10 \\
\hline & & Can & $662.3 \pm 204.3$ & $572.8 \pm 212.7$ & $-89.6 \pm 34.5$ & $<0.001^{* * *}$ & 9 \\
\hline & $15 \mathrm{~mm}$ & $\mathrm{BC}$ & $1,632.3 \pm 259.6$ & $1,596.3 \pm 241.6$ & $-36.0 \pm 69.0$ & 0.133 & 10 \\
\hline & & $\mathrm{LC}$ & $1,881.5 \pm 134.9$ & $1,793.9 \pm 197.1$ & $-87.6 \pm 113.0$ & $0.037^{*}$ & 10 \\
\hline & & Can & $517.2 \pm 240.0$ & $423.0 \pm 172.6$ & $-94.2 \pm 88.5$ & $0.013^{*}$ & 9 \\
\hline
\end{tabular}

Values are presented as mean \pm standard deviation.

HU, Hounsfield units; T1, before surgery; T2, after surgery; BC, buccal cortical bone; LC, lingual cortical bone; Can, cancellous bone.

${ }^{*} p<0.05,{ }^{* *} p<0.01,{ }^{* * *} p<0.001$.

The paired sample $t$-tests or ${ }^{\dagger}$ Wilcoxon signed rank tests were performed $(\alpha=0.05)$. 
cortical bone in both jaws (Tables 2 and 3).

The mean values of bone density in the extra-alveolar area were compared between T1 and T2 (Table 4). Statistically significant differences were found in Me and the cancellous bone of $\mathrm{C} 4$ in diagonal measurements $(p<0.05)$. The cancellous bone density of the condyle showed a large decrease, but it was not statistically significant. The Kruskal-Wallis test showed no difference in the bone density at different depths at the same site (Table 5).

A few sites showed statistically significant differences in bone density between patients who underwent genioplasty and those who did not. In the maxilla, premolar buccal cortical bone density at a depth of $10 \mathrm{~mm}$ and incisal lingual cortical bone density at a depth of $5 \mathrm{~mm}$ showed statistical significance. In the mandible, there was a significant difference in retromolar cancellous bone density at a depth of $5 \mathrm{~mm}$ and incisal lingual cortical bone density at a depth of $15 \mathrm{~mm}$. Among the extra-alveolar sites, the top of the head and cancellous bone density in the condyle showed a statistically significant difference between the two groups (Tables 6-8).

\section{DISCUSSION}

Liou et al. ${ }^{7}$ suggested that orthognathic surgery triggers a 3- to 4-month period of enhanced osteoclastic activity and metabolic changes in the alveolus postoperatively, which can possibly accelerate postoperative orthodontic tooth movement. The recent popularity of surgery-first orthognathic treatment plans could be partially due to the postsurgical acceleration of tooth movement and the resultant shorter duration of orthodontic treatment. ${ }^{11}$ Kang et al. ${ }^{12}$ showed this radiologically by using fractal analysis to observe trabecular changes in the mandible after orthognathic surgery. They found bone density reduction after orthognathic surgery. In our study, we found similar results using $\mathrm{HU}$

Table 4. Comparison of bone density (HU) in the extra-alveolar area between $\mathrm{T} 1$ and $\mathrm{T} 2$ measurements

\begin{tabular}{llccrc}
\hline & Site & T1 & T2 & $\Delta($ T2-T1) & $p$-value \\
\hline Menton & & $1,888.5 \pm 141.4$ & $1,843.7 \pm 139.9$ & $-44.8 \pm 48.7$ & $0.017^{*}$ \\
Top of head & & $1,730.2 \pm 118.5$ & $1,698.6 \pm 122.0$ & $-31.6 \pm 85.0$ & 0.270 \\
Condyle & Medial cortex & $872.7 \pm 269.3$ & $879.3 \pm 293.1$ & $6.6 \pm 99.4$ & 0.838 \\
& Lateral cortex & $887.9 \pm 233.6$ & $870.4 \pm 295.5$ & $-17.5 \pm 120.0$ & 0.656 \\
& Can & $230.9 \pm 69.6$ & $204.1 \pm 72.3$ & $-26.8 \pm 38.6$ & 0.056 \\
Vertebrae & ASC & $736.9 \pm 306.4$ & $766.3 \pm 265.7$ & $29.4 \pm 150.8$ & 0.553 \\
& PIC & $1,068.9 \pm 298.9$ & $1,050.3 \pm 318.9$ & $-18.6 \pm 129.8$ & 0.661 \\
& DCan & $371.9 \pm 94.3$ & $317.7 \pm 122.6$ & $-54.2 \pm 52.6$ & $0.010^{*}$ \\
\hline
\end{tabular}

Values are presented as mean \pm standard deviation.

The paired sample $t$-test $(\alpha=0.05)$ was performed.

HU, Hounsfield units; T1, before surgery; T2, after surgery; Can, cancellous bone; ASC, anterosuperior cortical bone; PIC, posteroinferior cortical bone; DCan, diagonal cancellous bone.

${ }^{*} p<0.05$.

Table 5. Comparison of alveolar bone density (HU) changes according to the depths

\begin{tabular}{|c|c|c|c|c|}
\hline \multicolumn{2}{|c|}{ Site } & \multirow{2}{*}{$\frac{\text { BC (p-value) }}{0.624}$} & \multirow{2}{*}{$\frac{\text { LC (p-value) }}{0.142}$} & \multirow{2}{*}{$\frac{\text { Can (p-value) }}{-}$} \\
\hline Maxilla & Tuberosity & & & \\
\hline & Molar & 0.107 & 0.571 & 0.285 \\
\hline & Premolar & 0.787 & 0.511 & 0.117 \\
\hline & Incisal & 0.401 & 0.904 & 0.295 \\
\hline \multirow[t]{4}{*}{ Mandible } & Retromolar pad & 0.345 & 0.337 & 0.823 \\
\hline & Molar & 0.582 & 0.243 & 0.166 \\
\hline & Premolar & 0.675 & 0.945 & 0.817 \\
\hline & Incisal & 0.377 & 0.148 & 0.859 \\
\hline
\end{tabular}

An independent-samples Kruskal-Wallis test was performed.

HU, Hounsfield units; BC, buccal cortical bone; LC, lingual cortical bone; Can, cancellous bone. 
Table 6. Comparison of pre- and postsurgical differences (T2-T1) in maxillary alveolar bone density (HU) between groups of patients who had undergone genioplasty and those who had not

\begin{tabular}{|c|c|c|c|c|c|}
\hline \multicolumn{3}{|c|}{ Site } & \multirow{2}{*}{$\begin{array}{c}\text { With G }(\mathbf{n}=\mathbf{6}) \\
-11.5 \pm 78.5\end{array}$} & \multirow{2}{*}{$\begin{array}{c}\text { Without G }(\mathbf{n}=\mathbf{4}) \\
-19.3 \pm 99.6\end{array}$} & \multirow{2}{*}{$\begin{array}{c}p \text {-value } \\
>0.999\end{array}$} \\
\hline Tuberosity & $5 \mathrm{~mm}$ & $\mathrm{BC}^{\dagger}$ & & & \\
\hline & & $\mathrm{LC}^{\dagger}$ & $-25.0 \pm 83.4$ & $-29.3 \pm 25.7$ & $>0.999$ \\
\hline & & $\mathrm{Can}^{\dagger}$ & $-66.5 \pm 33.2$ & $-109.3 \pm 70.3$ & 0.400 \\
\hline & $10 \mathrm{~mm}$ & $\mathrm{BC}^{\dagger}$ & $-62.5 \pm 33.2$ & $82.5 \pm 111.0$ & 0.333 \\
\hline & & $\mathrm{LC}^{\dagger}$ & $-69.0 \pm 5.7$ & $-71.0 \pm 19.8$ & $>0.999$ \\
\hline & & Can & - & - & - \\
\hline & $15 \mathrm{~mm}$ & $\mathrm{BC}$ & - & - & - \\
\hline & & $\mathrm{LC}$ & - & - & - \\
\hline & & Can & - & - & - \\
\hline \multirow[t]{9}{*}{ Molar } & $5 \mathrm{~mm}$ & $\mathrm{BC}$ & $-31.0 \pm 39.7$ & $6.5 \pm 33.3$ & 0.176 \\
\hline & & $\mathrm{LC}$ & $-8.3 \pm 69.2$ & $-44.2 \pm 64.2$ & 0.425 \\
\hline & & Can & $-46.3 \pm 81.5$ & $-70.0 \pm 70.5$ & 0.675 \\
\hline & $10 \mathrm{~mm}$ & $\mathrm{BC}$ & $2.0 \pm 35.8$ & $-179.5 \pm 135.9$ & $0.042^{*}$ \\
\hline & & $\mathrm{LC}$ & $-32.8 \pm 64.7$ & $-4.3 \pm 167.4$ & 0.758 \\
\hline & & $\mathrm{Can}^{\dagger}$ & 72.0 & -251.0 & $>0.999$ \\
\hline & $15 \mathrm{~mm}$ & $\mathrm{BC}^{\dagger}$ & $-45.0 \pm 63.6$ & $-114.0 \pm 83.4$ & 0.667 \\
\hline & & $\mathrm{LC}^{\dagger}$ & $23.5 \pm 33.2$ & $-11.3 \pm 105.4$ & $>0.999$ \\
\hline & & Can & 103.0 & - & - \\
\hline \multirow[t]{9}{*}{ Premolar } & $5 \mathrm{~mm}$ & $\mathrm{BC}$ & $-119.5 \pm 83.6$ & $73.5 \pm 83.2$ & $0.007^{* *}$ \\
\hline & & $\mathrm{LC}$ & $-34.8 \pm 51.2$ & $70.7 \pm 74.2$ & $0.040^{*}$ \\
\hline & & $\mathrm{Can}^{\dagger}$ & $-71.8 \pm 32.0$ & $-106.0 \pm 121.9$ & 0.064 \\
\hline & $10 \mathrm{~mm}$ & $\mathrm{BC}$ & $-19.8 \pm 70.7$ & $-29.7 \pm 66.0$ & 0.826 \\
\hline & & $\mathrm{LC}$ & $3.5 \pm 19.8$ & $37.8 \pm 154.4$ & 0.676 \\
\hline & & Can & $-112.3 \pm 63.1$ & $-56.0 \pm 24.6$ & 0.211 \\
\hline & $15 \mathrm{~mm}$ & $\mathrm{BC}$ & $-64.8 \pm 166.2$ & $-34.8 \pm 59.5$ & 0.746 \\
\hline & & $\mathrm{LC}$ & $-50.7 \pm 99.1$ & $-1.5 \pm 58.7$ & 0.444 \\
\hline & & Can & $-2.5 \pm 41.7$ & - & - \\
\hline \multirow[t]{9}{*}{ Incisal } & $5 \mathrm{~mm}$ & $\mathrm{BC}$ & $-4.5 \pm 130.6$ & $-63.3 \pm 60.2$ & 0.356 \\
\hline & & $\mathrm{LC}$ & $-147.5 \pm 61.7$ & $51.7 \pm 146.4$ & $0.035^{*}$ \\
\hline & & Can & $-176.3 \pm 88.4$ & $-79.8 \pm 154.7$ & 0.369 \\
\hline & $10 \mathrm{~mm}$ & $\mathrm{BC}$ & $-64.3 \pm 81.4$ & $43.8 \pm 128.8$ & 0.178 \\
\hline & & $\mathrm{LC}$ & $-57.5 \pm 90.6$ & $-14.0 \pm 175.0$ & 0.663 \\
\hline & & Can & $-122.3 \pm 95.7$ & $-62.3 \pm 133.4$ & 0.515 \\
\hline & $15 \mathrm{~mm}$ & $\mathrm{BC}$ & $-99.0 \pm 75.1$ & $-17.7 \pm 47.5$ & 0.066 \\
\hline & & $\mathrm{LC}$ & $-22.0 \pm 15.4$ & $-44.3 \pm 88.6$ & 0.638 \\
\hline & & Can & $-12.3 \pm 35.2$ & $-54.5 \pm 36.5$ & 0.143 \\
\hline
\end{tabular}

Values are presented as mean \pm standard deviation or number only when only one measurement was possible.

Some measurements could not be obtained due to adjacent anatomic structures (i.e., third molar, maxillary sinus) or other objects (i.e., orthodontic miniscrew).

HU, Hounsfield units; T1, before surgery; T2, after surgery; With G, patients with genioplasty; Without G, patients without genioplasty; BC, buccal cortical bone; LC, lingual cortical bone; Can, cancellous bone.

${ }^{*} p<0.05,{ }^{* *} p<0.01$.

The independent sample $t$-tests or ${ }^{\dagger}$ Mann-Whitney tests were performed. 
Table 7. Comparison of pre- and postsurgical differences (T2-T1) in mandibular alveolar bone density (HU) between groups of patients who had undergone genioplasty and those who had not

\begin{tabular}{|c|c|c|c|c|c|}
\hline \multicolumn{3}{|c|}{ Site } & \multirow{2}{*}{$\begin{array}{c}\text { With G }(\mathbf{n}=\mathbf{6}) \\
-33.0 \pm 77.4\end{array}$} & \multirow{2}{*}{$\begin{array}{c}\text { Without G }(\mathbf{n}=\mathbf{4}) \\
-69.8 \pm 107.9\end{array}$} & \multirow{2}{*}{$\frac{p \text {-value }}{0.575}$} \\
\hline Retromolar pad & $5 \mathrm{~mm}$ & $\mathrm{BC}$ & & & \\
\hline & & $\mathrm{LC}^{\dagger}$ & $-35.5 \pm 20.7$ & $-61.2 \pm 45.6$ & 0.114 \\
\hline & & Can & $5.3 \pm 80.9$ & $-117.3 \pm 61.8$ & $0.026^{*}$ \\
\hline & $10 \mathrm{~mm}$ & $\mathrm{BC}$ & $-108.8 \pm 149.7$ & $-59.7 \pm 124.2$ & 0.587 \\
\hline & & LC & $-91.0 \pm 80.0$ & $-102.3 \pm 134.7$ & 0.885 \\
\hline & & Can & $-52.8 \pm 41.1$ & $-82.3 \pm 67.3$ & 0.459 \\
\hline & $15 \mathrm{~mm}$ & $\mathrm{BC}$ & $-11.0 \pm 13.9$ & $-18.5 \pm 41.6$ & 0.696 \\
\hline & & $\mathrm{LC}^{\dagger}$ & $-13.5 \pm 22.0$ & $-30.7 \pm 111.2$ & 0.610 \\
\hline & & Can & $-36.8 \pm 35.7$ & $-70.7 \pm 75.4$ & 0.432 \\
\hline \multirow[t]{9}{*}{ Molar } & $5 \mathrm{~mm}$ & $\mathrm{BC}$ & $-48.0 \pm 45.0$ & $-24.5 \pm 87.1$ & 0.637 \\
\hline & & $\mathrm{LC}$ & $-72.8 \pm 75.3$ & $-90.0 \pm 65.3$ & 0.710 \\
\hline & & Can & $-141.8 \pm 89.8$ & $-70.8 \pm 46.2$ & 0.135 \\
\hline & $10 \mathrm{~mm}$ & $\mathrm{BC}$ & $-95.0 \pm 119.3$ & $-70.0 \pm 67.4$ & 0.680 \\
\hline & & $\mathrm{LC}^{\dagger}$ & $-70.3 \pm 117.5$ & $-36.2 \pm 52.9$ & $>0.999$ \\
\hline & & $\mathrm{Can}^{\dagger}$ & $-80.8 \pm 84.6$ & $-75.8 \pm 72.4$ & $>0.999$ \\
\hline & $15 \mathrm{~mm}$ & $\mathrm{BC}$ & $-105.5 \pm 159.5$ & $-39.8 \pm 52.3$ & 0.366 \\
\hline & & LC & $-36.0 \pm 47.5$ & $-40.3 \pm 73.8$ & 0.920 \\
\hline & & Can & $-52.8 \pm 21.6$ & $-32.3 \pm 34.7$ & 0.285 \\
\hline \multirow[t]{9}{*}{ Premolar } & $5 \mathrm{~mm}$ & $\mathrm{BC}$ & $-68.5 \pm 68.5$ & $-64.8 \pm 48.7$ & 0.923 \\
\hline & & LC & $-74.8 \pm 70.2$ & $-89.5 \pm 105.3$ & 0.813 \\
\hline & & Can & $-23.0 \pm 16.2$ & $-70.5 \pm 53.0$ & 0.126 \\
\hline & $10 \mathrm{~mm}$ & $\mathrm{BC}$ & $-41.5 \pm 80.2$ & $-20.7 \pm 215.6$ & 0.860 \\
\hline & & $\mathrm{LC}^{\dagger}$ & $-87.0 \pm 70.9$ & $-79.0 \pm 129.8$ & 0.610 \\
\hline & & Can & $-24.8 \pm 32.9$ & $-108.0 \pm 99.9$ & 0.104 \\
\hline & $15 \mathrm{~mm}$ & $\mathrm{BC}$ & $-122.5 \pm 146.6$ & $-56.8 \pm 60.6$ & 0.347 \\
\hline & & LC & $-133.3 \pm 146.8$ & $-41.7 \pm 28.2$ & 0.302 \\
\hline & & Can & $-57.5 \pm 33.8$ & $-72.8 \pm 61.3$ & 0.664 \\
\hline \multirow[t]{9}{*}{ Incisal } & $5 \mathrm{~mm}$ & $\mathrm{BC}$ & $-67.0 \pm 50.0$ & $-40.5 \pm 61.6$ & 0.496 \\
\hline & & $\mathrm{LC}$ & $-72.8 \pm 88.1$ & $-62.7 \pm 99.6$ & 0.874 \\
\hline & & Can & $-142.3 \pm 96.2$ & $-76.3 \pm 45.6$ & 0.177 \\
\hline & $10 \mathrm{~mm}$ & $\mathrm{BC}$ & $-41.5 \pm 38.6$ & $7.0 \pm 52.6$ & 0.155 \\
\hline & & LC & $-21.0 \pm 33.4$ & $-16.7 \pm 43.5$ & 0.871 \\
\hline & & $\mathrm{Can}^{+}$ & $-94.7 \pm 27.4$ & $-87.0 \pm 39.8$ & 0.548 \\
\hline & $15 \mathrm{~mm}$ & $\mathrm{BC}$ & $-53.5 \pm 111.6$ & $-24.3 \pm 26.2$ & 0.641 \\
\hline & & LC & $-180.8 \pm 111.8$ & $-25.5 \pm 62.6$ & $0.022^{*}$ \\
\hline & & Can & $-155.0 \pm 132.9$ & $-63.8 \pm 46.2$ & 0.156 \\
\hline
\end{tabular}

Values are presented as mean \pm standard deviation.

HU, Hounsfield units; T1, before surgery; T2, after surgery; With G, patients with genioplasty; Without G, patients without genioplasty; BC, buccal cortical bone; LC, lingual cortical bone; Can, cancellous bone.

${ }^{*} p<0.05$.

The independent sample $t$-tests or ${ }^{\dagger}$ Mann-Whitney tests were performed. 
Table 8. Comparison of pre- and postsurgical differences (T2-T1) in extra-alveolar bone density (HU) between groups of patients who had undergone genioplasty and those who had not

\begin{tabular}{|c|c|c|c|c|}
\hline \multicolumn{2}{|c|}{ Site } & \multirow{2}{*}{$\begin{array}{c}\text { With G }(\mathbf{n}=\mathbf{6}) \\
-75.3 \pm 67.5\end{array}$} & \multirow{2}{*}{$\begin{array}{c}\text { Without G }(\mathbf{n}=\mathbf{4}) \\
-24.5 \pm 17.2\end{array}$} & \multirow{2}{*}{$\frac{p \text {-value }}{0.230}$} \\
\hline Menton & & & & \\
\hline Top of head & & $-97.0 \pm 99.6$ & $12.0 \pm 36.6$ & $0.037^{*}$ \\
\hline \multirow[t]{3}{*}{ Condyle } & Medial cortex $^{\dagger}$ & $-37.5 \pm 55.9$ & $36.0 \pm 115.4$ & 0.352 \\
\hline & Lateral cortex $^{\dagger}$ & $-56.5 \pm 49.8$ & $8.5 \pm 149.7$ & 0.476 \\
\hline & Can & $-58.3 \pm 39.1$ & $-5.8 \pm 21.2$ & $0.024^{*}$ \\
\hline \multirow[t]{3}{*}{ Vertebrae } & ASC & $5.8 \pm 84.2$ & $45.2 \pm 189.5$ & 0.710 \\
\hline & PIC & $-65.5 \pm 72.8$ & $12.7 \pm 155.5$ & 0.382 \\
\hline & DCan $^{\dagger}$ & $-83.5 \pm 49.7$ & $-34.7 \pm 48.5$ & 0.114 \\
\hline
\end{tabular}

Values are presented as mean \pm standard deviation.

HU, Hounsfield units; T1, before surgery; T2, after surgery; With G, patients with genioplasty; Without G, patients without genioplasty; Can, cancellous bone; ASC, anterosuperior cortical bone; PIC, posteroinferior cortical bone; DCan, diagonal cancellous bone.

${ }^{*} p<0.05$.

The independent sample $t$-tests or ${ }^{\dagger}$ Mann-Whitney tests were performed.

values obtained with $\mathrm{CT}$, which is regarded as one of the established methods for determining bone density. ${ }^{13}$

In addition, we found that a decrease in bone density was more distinct in cancellous bone than in cortical bone in both the alveolar and extra-alveolar areas. It can be assumed this was based on a difference in the turnover rate of cancellous bone vs. cortical bone. Cancellous bone is generally considered to show a higher turnover than cortical bone, so the effect of RAP will be less intense in cortical bone. ${ }^{14}$ Moreover, SAP is thought to be prominent in cancellous bone as well. Mueller et al. ${ }^{6}$ claimed that SAP occurred only in the cancellous bone because SAP was induced by the humoral factors that act systemically. Later, in 2009, Funk et al. ${ }^{15}$ reported the effect of SAP in cortical bone, observing a systemic anabolic effect on cortical bone caused by distraction osteogenesis. The differences between these findings were attributed to the choice of methods.

In the present study, the bone density decrease was more evident in the mandible than in the maxilla. This finding contrasts with the expected results based on the previous studies, which included a higher rate of resorption and consequently faster tooth movement in the maxilla. ${ }^{16,17}$ However, Huja et al. ${ }^{18}$ reported that trabecular bone remodeling was almost double in the mandible than that in the maxilla, and Kotze et al. ${ }^{19}$ reported much faster recovery of bone density in the mandible than the maxilla and suggested that the denser environment provided more regeneration tissue and bone cells.

This study showed no statistically significant differences in the depths of the sites measured. It could be hypothesized that the orthognathic surgery was extensively invasive enough to impact and facilitate the bone turnover mechanism over the entire depth; however, further research on a larger sample will be essential for accurate interpretation.

$\mathrm{McBride}$ et $\mathrm{al}^{20}{ }^{20}$ reported that the extent of the surgical insult was related to the degree of reduction in the postoperative bone density. In our study, the patients who underwent genioplasty and those who did not showed no statistical difference at most sites. We could not find any order or trends, so we suspected that the IVRO and Le Fort 1 osteotomy were invasive enough to reduce the effect of genioplasty. This could explain the evidence for SAP, where a systemic effect was potentiated by the surgeries performed.

In the present study, the postoperative CTs were taken $5.8 \pm 2.6$ days after the surgery. Mueller et al. ${ }^{6}$ reported that the evidence of RAP was first observed after 10 days of healing, and there was almost complete recovery after 120 days in rats. Some studies also suggested that RAP in humans begins within a few days after surgery and typically peaks in the first or second month thereafter, while it might take six to more than 24 months for it to subside. $^{21,22}$ Therefore, in contrast to present research, CT scans taken with an interval of two months after surgery can be more discernible. However, Liou et al. $^{7}$ reported that the highest level of C-terminal telopeptide of type 1 collagen, a biomarker for osteoclastic activity, and nearly the highest level of mobility in the teeth occurs in the first week following orthognathic surgery. This was in accordance with the timing when the CT scans were taken in our study and explains the observations.

In this study, we aimed to facilitate a better understanding of RAP and SAP following orthognathic surgery. According to Sebaoun et al., ${ }^{23}$ the range of RAP rarely extends more than the distance of one tooth. Taking this into consideration, Le Fort 1 osteotomy should 
be sufficient to cause RAP in the whole maxillary arch, but IVRO or sagittal split ramus osteotomy might not evoke RAP in the anterior region of the mandible by itself as the surgical field is limited to the mandibular posterior area distal to the first molar.

Although genioplasty also could cause RAP in the areas of Me and mandibular anterior alveolar bone, the bone densities in these areas decreased even without genioplasty and there was no statistical difference in bone density between the patients who underwent genioplasty and those who did not. This could explain the relationship between IVRO and exhibition of SAP. Furthermore, the decrease in the cancellous bone density of the cervical vertebra $\mathrm{e}^{24}$ after orthognathic surgery can be considered as evidence of the presence of SAP. Therefore, SAP induced by IVRO can contribute to accelerated postsurgical orthodontic tooth movement.

The bone density decrease that occurred after orthognathic surgery in this study might not suffice as direct evidence that RAP and SAP occur after orthognathic surgery. However, the rate of tooth movement is inversely related to the bone density ${ }^{16}$ and the bone density decrease could contribute to faster orthodontic tooth movement in postoperative orthodontic treatment.

The main limitation of this study was the small sample size. Cases with CT images were chosen for a more precise evaluation of bone density. The high radiation doses associated with CT imaging meant that CT images of every orthognathic patient could not be taken. To further investigate this issue, more samples would be required. To expand the research in this field, a proper measurement method should be developed using conebeam computed tomography, a commonly used modality in dentistry.

\section{CONCLUSION}

Pre- and postsurgical bone densities were measured and compared in alveolar and extra-alveolar sites.

1. Overall alveolar bone density tends to decrease. In particular, there was a statistically significant $(p<0.05)$ decrease in most alveolar sites in the mandible.

2. Cancellous bone showed greater bone density decrease than did cortical bone. There was a statistically significant $(p<0.05)$ decrease in bone density of the cancellous bone in most sites.

3. There was no statistical difference in bone density in the alveolar site with regard to depth apically from the alveolar crest.

4. There was almost no statistically significant difference in bone density between groups with and without genioplasty, suggesting the evidence of SAP.

\section{CONFLICTS OF INTEREST}

No potential conflict of interest relevant to this article was reported.

\section{ACKNOWLEDGEMENTS}

This paper was supported by Wonkwang University in 2020.

\section{REFERENCES}

1. Köle H. Surgical operations on the alveolar ridge to correct occlusal abnormalities. Oral Surg Oral Med Oral Pathol 1959;12:515-29.

2. Frost HM. The regional accelerated phenomenon. Orthop Clin North Am 1981;12:725-6.

3. Frost HM. The regional acceleratory phenomenon: a review. Henry Ford Hosp Med J 1983;31:3-9.

4. Frost HM. The biology of fracture healing. An overview for clinicians. Part 1. Clin Orthop Relat Res 1989;(248):283-93.

5. Melsen B. Tissue reaction to orthodontic tooth movement--a new paradigm. Eur J Orthod 2001;23: 671-81.

6. Mueller M, Schilling T, Minne HW, Ziegler R. A systemic acceleratory phenomenon (SAP) accompanies the regional acceleratory phenomenon (RAP) during healing of a bone defect in the rat. J Bone Miner Res 1991;6:401-10.

7. Liou EJ, Chen PH, Wang YC, Yu CC, Huang CS, Chen YR. Surgery-first accelerated orthognathic surgery: postoperative rapid orthodontic tooth movement. J Oral Maxillofac Surg 2011;69:781-5.

8. Verna C, Zaffe D, Siciliani G. Histomorphometric study of bone reactions during orthodontic tooth movement in rats. Bone 1999;24:371-9.

9. Melsen B. Biological reaction of alveolar bone to orthodontic tooth movement. Angle Orthod 1999; 69:151-8.

10. White SC, Rudolph DJ. Alterations of the trabecular pattern of the jaws in patients with osteoporosis. Oral Surg Oral Med Oral Pathol Oral Radiol Endod 1999;88:628-35.

11. Peiró-Guijarro MA, Guijarro-Martínez R, HernándezAlfaro F. Surgery first in orthognathic surgery: a systematic review of the literature. Am J Orthod Dentofacial Orthop 2016;149:448-62.

12. Kang HJ, Jeong SW, Jo BH, Kim YD, Kim SS. Observation of trabecular changes of the mandible after orthognathic surgery using fractal analysis. J Korean Assoc Oral Maxillofac Surg 2012;38:96-100.

13. Shahlaie M, Gantes B, Schulz E, Riggs M, Crigger M. Bone density assessments of dental implant sites: 1 . 
Quantitative computed tomography. Int J Oral Maxillofac Implants 2003;18:224-31.

14. Parfitt AM. Misconceptions (2): turnover is always higher in cancellous than in cortical bone. Bone 2002;30:807-9.

15. Funk JF, Krummrey G, Perka C, Raschke MJ, Bail HJ. Distraction osteogenesis enhances remodeling of remote bones of the skeleton: a pilot study. Clin Orthop Relat Res 2009;467:3199-205.

16. Roberts WE. Bone physiology, metabolism and biomechanics in orthodontic practice. In: Graber TM, Vanarsdall RL Jr, Vig KWL, eds. Orthodontics: current principles and techniques. 4th ed. St Louis: Mosby-Year Book; 2005. p. 221-92.

17. Deguchi T, Takano-Yamamoto T, Yabuuchi T, Ando R, Roberts WE, Garetto LP. Histomorphometric evaluation of alveolar bone turnover between the maxilla and the mandible during experimental tooth movement in dogs. Am J Orthod Dentofacial Orthop 2008;133:889-97.

18. Huja SS, Fernandez SA, Hill KJ, Li Y. Remodeling dynamics in the alveolar process in skeletally mature dogs. Anat Rec A Discov Mol Cell Evol Biol 2006;288:1243-9.

19. Kotze MJ, Bütow KW, Olorunju SA, Kotze HF. A comparison of mandibular and maxillary alveolar osteogenesis over six weeks: a radiological examination. Head Face Med 2014;10:50.

20. McBride MD, Campbell PM, Opperman LA, Dechow $\mathrm{PC}$, Buschang $\mathrm{PH}$. How does the amount of surgical insult affect bone around moving teeth? Am J Orthod Dentofacial Orthop 2014;145(4 Suppl):S92-9.

21. Kausal S, Agrawal A, Misal AN, Toshniwal NG. Accelerted orthodontic tooth movement: a new paradigm in orthodontics. Int J Oral Health Med Res 2015;2:94-6.

22. Schilling T, Müller M, Minne HW, Ziegler R. Influence of inflammation-mediated osteopenia on the regional acceleratory phenomenon and the systemic acceleratory phenomenon during healing of a bone defect in the rat. Calcif Tissue lnt 1998;63:160-6.

23. Sebaoun JD, Kantarci A, Turner JW, Carvalho RS, Van Dyke TE, Ferguson DJ. Modeling of trabecular bone and lamina dura following selective alveolar decortication in rats. J Periodontol 2008;79:167988.

24. Zhang Y, Zhou Z, Wu C, Zhao D, Wang C, Cheng X, et al. Population-stratified analysis of bone mineral density distribution in cervical and lumbar vertebrae of Chinese from quantitative computed tomography. Korean J Radiol 2016;17:581-9. 